\title{
Differences among Three Branded Formulations of Hyaluronic Acid: Data from Environmental Scanning Electron Microscope Profile, Rheology Behavior and Biological Activity
}

\author{
Mantovani Veronica ${ }^{1}$, Galeotti Fabio ${ }^{1}$, Volpi Nicola ${ }^{1}$, Pozzi Paolo ${ }^{2}$ and Baraldi Enrica ${ }^{3 *}$ \\ ${ }^{1}$ Department of Life Sciences, University of Modena and Reggio Emilia, Italy \\ ${ }^{2}$ Department of Engineering, University of Modena and Reggio Emilia, Italy \\ ${ }^{3}$ Chiesi Famaceutici S.p.A., Medical Department, Parma, Italy \\ *Corresponding author: Enrica Baraldi, Chiesi Farmaceutici S.p.A., Via Palermo 26/A, 43122 Parma, Italy
}

\section{ARTICLE INFO}

Received: April 01, 2019

Published: April 12, 2019

Citation: Mantovani V, Galeotti F, Volpi N, Pozzi P, Baraldi E. Differences among Three Branded Formulations of Hyaluronic Acid: Data from Environmental Scanning Electron Microscope Profile, Rheology Behavior and Biological Activity. Biomed J Sci \& Tech Res 17(1)-2019. BJSTR. MS.ID.002938.

Keywords: Hyaluronic Acid; Formulations; Viscosupplementation; Esem; Rheology

\section{ABSTRACT}

Background: This study has analysed the viscosupplemental proprieties of three commercially available formulations of Hyaluronic Acid (HA) suspension (F1: Synvisc, Hylan G-F 20; F2: Hyalgan; F3: Donegal HA 2.0), which differ in composition, Molecular Weight (MW) and HA content.

Methods: Analyses were conducted using rheology measurements and Environmental Scanning Electron Microscope (ESEM). The capacity of the three tested formulations to inhibit specific Metalloproteases (MMPs) was also evaluated.

Results: F1 is the only sample showing viscoelastic properties but may have increased immunogenicity attributable to the subsequent chemical cross-linking process that enhances the MW. F2 and F3 show a lower viscosity compared to F1. F2 has the lowest viscosity at low shear rate, the lower independence from the oscillatory stress and a solution-like rheology behaviour. F3 display a solution behaviour. However, unlike F2, F3 crossover point falls in the middle of the frequency range of interest showing a considerable rheological behaviour. The internal structure of F3 (pseudo-spongy thick filaments) suggests that it has the ability to interact with a great water content. The crossover points of the examined samples clearly reveal their different rheological behaviour, allowing their classification in gel-like or solution-like materials. F3 has higher ability in inhibiting MMP2 and MMP-9 activity compared to F1 and F2, probably due to its specific MW and/or higher HA concentration.

Conclusion: The three tested HA formulations differ in rheological properties and inhibition of MMP-2 and MMP-9 activity. F3 seems to be the most appropriate formulation for the treatment of osteoarthritis and rheumatoid arthritis.

\section{Introduction}

Hyaluronic Acid (HA), or hyaluronan, is a polysaccharide composed of a repeated disaccharide unit of $\beta-1,4-D$-glucuronic acid - $\beta-1,3-N$-acetyl-D-glucosamine linked by glycosidic bonds. $\mathrm{HA}$ is the simplest member of a group of macromolecules known as Glycosaminoglycans (GAGs). As the only GAG, it is not covalently associated with a core protein, not synthesized through the Golgi pathway, and the only non-sulphated one. HA can be found in skin, joints, eyes and most other organs and tissues. It was originally discovered in the vitreous body of the eye and subsequently it was identified in most parts of the body, including the Extracellular Matrix (ECM) of cartilage tissues [1]. The molecular weight of HA can range as high as several million Daltons [2]. Hyaluronic acid is an essential component of the ECM, in which its structural and 
biological properties account for its activity in morphogenesis, wound repair and tissue organisation [3,4]. Additionally, HA is rapidly turned over in the body by hyaluronidases enzymatic degradation, with consequent relatively short tissue half-lives [5]. The relevance of HA in living organisms and in artificial biological systems has been emphasized and reported in many occasions [6]. Its most important physicochemical properties are its capacity to retain water, a very high hydration ratio, and its viscoelasticity. These properties contribute to the control of tissue hydration, permeability to small or large molecules and in several signaling pathways.

This polysaccharide displays particular conformational features, in fact, the macromolecular properties of high molecular weight HA in dilute solution have been interpreted on the basis of a worm-like coil with moderate stiffness [7] and more recently of a stiffish coil [8]. In physiological conditions, the conformation of the HA molecule is stiffened by a combination of the chemical structure, the internal hydrogen bonds, and the interactions with the solvent, which results in a coil structure that traps approximately 1000 times its weight in water. High concentrations of HA in physiological state lead to stable superstructures [9]. Increases in temperature determine a breaking of certain cooperative bonds, with consequent changes of HA rheological properties and induction of a rapid change of the HA superstructure from a long- to a restricted-connectivity water structuring [9]. Finally, HA macromolecular chains are not homogeneous in length, and consequently in molar mass, but reveal a certain extent of polydispersity [10]. Solutions of HA exhibit peculiar rheological properties beyond the lubricious and very hydrophilic function [11] When examined in the solution state, the HA polymer chains fit each other at very low concentrations, which further contribute to the unusual rheological characteristics. Above the entanglement point the viscosity increases rapidly and exponentially with concentration $\left(\sim \mathrm{c}^{3.3}\right)[12]$ and a solution of 10 $\mathrm{g} / \mathrm{l}$ may have a viscosity at low shear of $\sim 10^{6}$ times the viscosity of the solvent, whereas the viscosity may drop as much as $\sim 10^{3}$ times at high shear [13]. The elasticity of the system increases at high molecular weight and concentration of HA as expected for a molecular network.

A $1 \%$ solution is like jelly, but when put under pressure it moves easily and can be administered through a small-bore needle, acting as a "pseudo-plastic" material. The rheological properties of HA solutions allows an ideal use as a lubricant [6-15]. Hyaluronic acid and its derivatives have been used in medicines for may years [16]. In recent years, HA has been developed for the creation of new biomaterials for use in tissue engineering and regenerative medicine
[17] due to its biological properties and its lack of immunogenic and thrombogenic effects [18]. Solutions of HA do not have long lasting mechanical integrity $[19,20]$. Chemical modification and controlled covalent crosslinking have been necessary to generate HA-based hydrogels with desired mechanical properties, while at the same time maintaining their native biological functions. The modification of the molecular weight of HA, the extent of this modification and the concentration of the reactive HA precursors, hydrogels with varying stiffness, pore size and degradation rate can be rapidly realised. Further biological functions can be obtained by coupling the HA gels with different biological substances, cytokines and therapeutic drugs [21]. Traditional HA-based hydrogels are macroscopic networks made of randomly interconnected HA chains, but without the structural complexity and functional diversity of the natural ECM.

Drug molecules encapsulated in the network without any covalent linkage or other specific interactions may be released rapidly due to the relatively large pore size. If the crosslinking reaction takes place in a microscopic reaction vessel, HA hydrogel particles (HGPs, microgels or nanogels) can be produced [22]. Among the use of HA in clinical practice, the Intra-Articular (IA) administration is able to restore the normal viscoelastic properties of the pathologically altered Synovial Fluid (SF), which explains the term of the approach "viscosupplementation", and to protect the articular cartilage and soft tissue against mechanical injuries during joint movement [23]. It is thought that HA temporarily restores the lubricating and shock-absorbing effects of SF. Moreover, several studies suggest that viscosupplements also have disease modifying effects, such as reduction of synovial inflammation, [24] protection against cartilage erosion, [25] and promotion of SF endogenous HA production [26] which explains the term of the approach "viscoinduction". Here, we analyzed by Environmental Scanning Electron Microscope (ESEM) and rheology measurements, three different commercially available samples of HA suspension in order to monitor and compare their viscosupplemental proprieties. Finally, their capacity to inhibit specific Matrix Metalloproteases (MMPs) was also evaluated.

\section{Materials and Methods}

\section{Materials}

Hyaluronic acid samples in finished formulations were from three different producers, formulation 1 (Synvisc, Hylan G-F 20, Sanofi) prepared by using cross-linked HA, formulation 2 (Hyalgan, Fidia Farmaceutici) and formulation 3 (Donegal HA 2.0, Chiesi Farmaceutici). Table 1 shows the composition and properties of the three studied HA formulations.

Table 1: Composition and properties of the three products studied.

\begin{tabular}{|c|c|c|c|c|}
\hline HA Formulation & Bioactive molecule & Molecular Weight (MW) & HA Content & HA Origin \\
\hline 1 (Synvisc) & Hylan G-F 20 & $\begin{array}{c}\text { Hylan A has a MW }=\sim 6000 \\
\text { kDa and Hylan B is a } \\
\text { hydrogel }\end{array}$ & $8.0 \pm 2.0 \mathrm{mg} / \mathrm{mL}$ & Extractive \\
\hline 2 (Hyalgan) & HA sodium salt & $500-730 \mathrm{kDa}$ & $10 \mathrm{mg} / \mathrm{mL}$ & Extractive \\
\hline 3 (Donegal HA 2.0) & HA sodium salt & $800-1200 \mathrm{kDa}$ & $20 \mathrm{mg} / \mathrm{mL}$ & Fermentative \\
\hline
\end{tabular}




\section{ESEM Analysis}

Before analysis, the three studied HA samples were lyophilized and covered with a thin gold layer, metallization, to obtain images with higher resolution. The analyses were performed with a Scanning Electron Microscope Quanta ESEM-200 of Fei Company (Oxford Instruments) equipped with a: Secondary Electron Detector (SED) [High Vacuum], Secondary Electron Detector (LFD) [Low Vacuum], Fire secondary electrons (GSED) [ESEM mode], Backscattered Electron Detector (BSE) [High Vacuum and Low Vacuum], simultaneous detector of secondary electrons and backscattered (GBSD) [ESEM mode], backscattered detector (GAD) optimized for EDS analysis [Low-Vacuum mode], Table Peltier to cool the sample $\left[-25 /+55^{\circ} \mathrm{C}\right]$, Hot-Stage dynamic experiments at variable temperature $\left[\geq 1500^{\circ} \mathrm{C}\right]$, system for microanalysis X-EDS (Oxford INCA-350), Detector Si (Li) with thin window for investigation of elements with low atomic number, software and hardware for qualitative and quantitative analysis.

After covering with a thin gold layer (metallization) to obtain images having higher resolution, HA samples were analyzed to ESEM. Samples from various producers were freeze-dried before metallization shoving as a consequence a very low water content. Furthermore, HA surfaces were observed to be covered of saline in a more or less content.

\section{Rheology Measurements}

Rheological measurements were performed with a Thermo Haake Rheostress RS100, equipped with a cone-and-plate fixture consisting of a $1^{\circ}, 6 \mathrm{~cm}$ diameter stainless steel cone. The rheometer is equipped with a Thermo DC30 water bath Haake to regulate the sample temperature, a $2 \mathrm{ml}$ fluid sample was required and the rheology behaviour was investigated at $25^{\circ} \mathrm{C}$, and $37^{\circ} \mathrm{C}$. In all $\mathrm{HA}$ solution, an initial stress sweeps with an oscillatory stress, at a constant frequency of $\omega=1 \mathrm{~Hz}$, was applied to determine the region of linear response of the sample. On this basis, a value of applied stress within the linear regime was used in subsequent frequency sweeps. Steady shear flow experiments were carried out for shear rates up to $10^{3} \mathrm{~s}^{-1}$, and mechanical properties were investigated as a function of time by creep and recovery measurements.

\section{Inhibition of MMP-2 and MMP-9 Activity}

Blood samples were taken from healthy volunteers and serum samples were obtained in tubes with no additives. Methods of samples' collection, tracking, transportation and storage were in conformity with rules in force in Italy. An amount of $35 \mu \mathrm{g}$ of total HA from the three samples were added to previously separated serum and incubated at $37^{\circ} \mathrm{C}$ for $30 \mathrm{~min}$. After centrifugation at $1500 \mathrm{rpm}$ for $15 \mathrm{~min}$ at $4^{\circ} \mathrm{C}$, aliquots containing $140 \mu \mathrm{g}$ of proteins were analyzed by gelatine zymography carried out on $7.5 \%$ polyacrylamide gels copolymerized with $2 \mathrm{~g} / \mathrm{L}$ type A gelatine from porcine skin (Sigma-Aldrich). MMP calibrators were from capillary whole blood. Gelatin zymography (for both gelatinases, MMP-2 and -9) is one of the most commonly used tests to assess MMP activity. ProMMP-2 at $72 \mathrm{kDa}$, proMMP-9 and MMP-9 complexed forms at 92 and $225 \mathrm{kDa}$ were evaluated. Briefly, after electrophoresis, gels were washed in Triton X-100 (25 mL/L) and incubated for $24 \mathrm{~h}$ at $37^{\circ} \mathrm{C}$ in enzyme incubation buffer. Gel staining was performed in Coomassie brilliant blue R-250 (2 g/L) and destained in methanolacetic acid standard solution. Clear gelatinolytic bands on uniform blue background were densitometrically measured by a common image analyzer.

\section{Results}

\section{ESEM Profile}
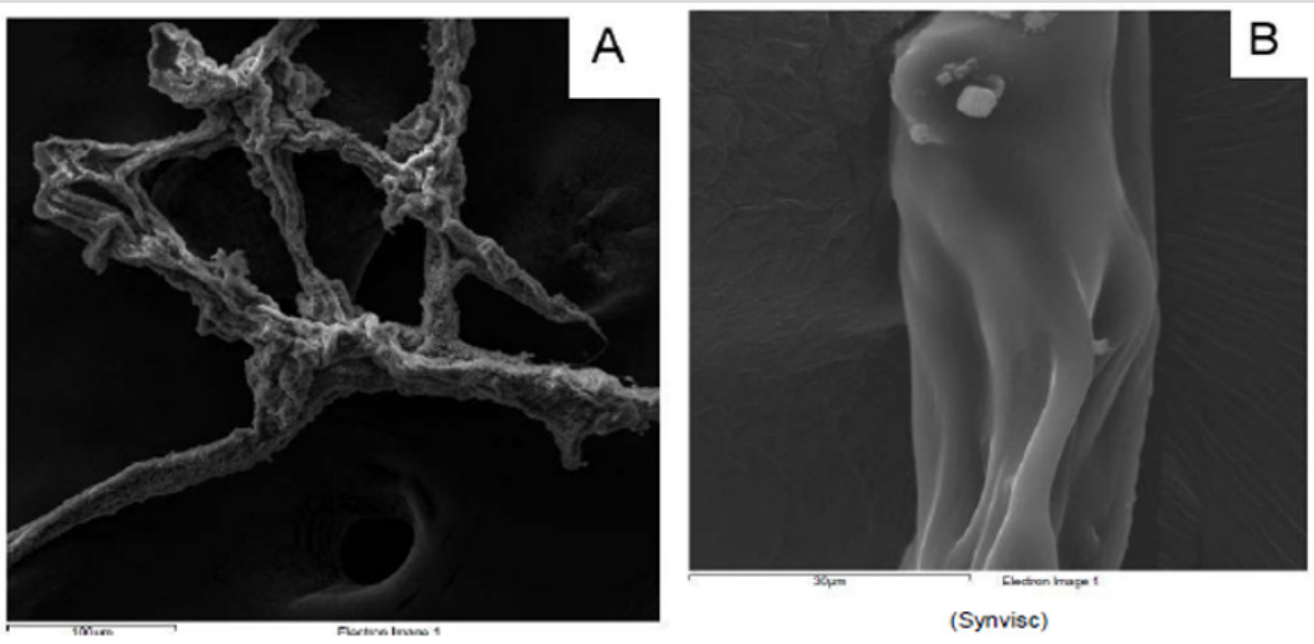

Figure 1: ESEM profile of HA from formulation 1 at different magnification (A. $100 \mu \mathrm{m}$ and B. $30 \mu \mathrm{m})$.

From a macroscopic point of view, HA formulation 1 shows a filamentous structure (Figure 1A) that tends to flake easily when subjected to mechanical stress. The substructure is characterized by a dense accumulation of filaments which, however, tend to be distributed side by side in longitudinal mode (Figure 1B). At macroscopic level, HA formulation 2 shows a collected structure of globular type (Figure 2A). By increasing the magnification, it can be seen that the HA substructure is characterized by a dense network 
of filaments which tend to clump together in a compact manner without a clear and repeated organization (Figure 2B). Finally, $\mathrm{HA}$ formulation 3 is characterized by a very compact structure with defined profiles showing at macroscopic level a larger size compared to the other analyzed samples (Figure 3A). The internal structure of this globular bodies is made of pseudo-spongy thick filaments where HA is distributed in a compact branched nature (Figure 3B). This structure suggests that this macromolecule has the strong ability to interact with a great content of water.
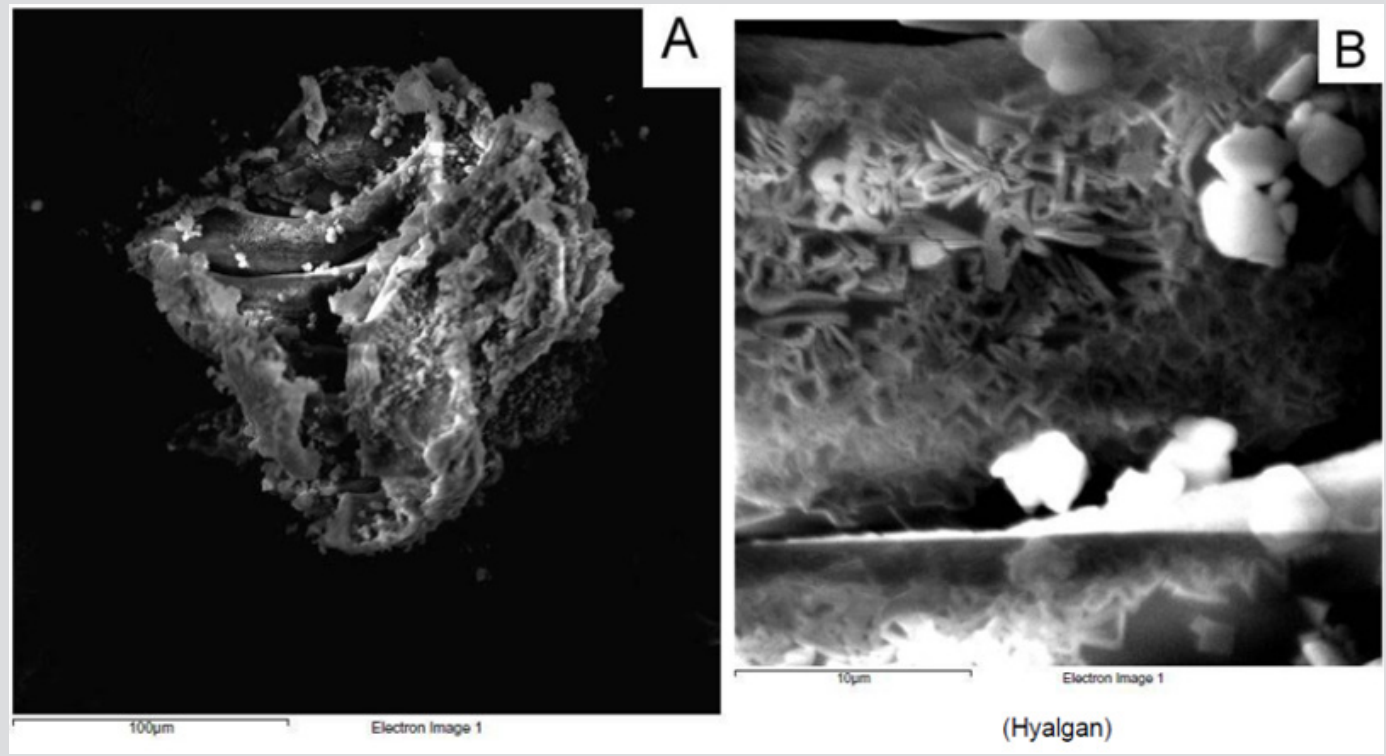

Figure 2: ESEM acquisition of HA from formulation 2 at different magnification.
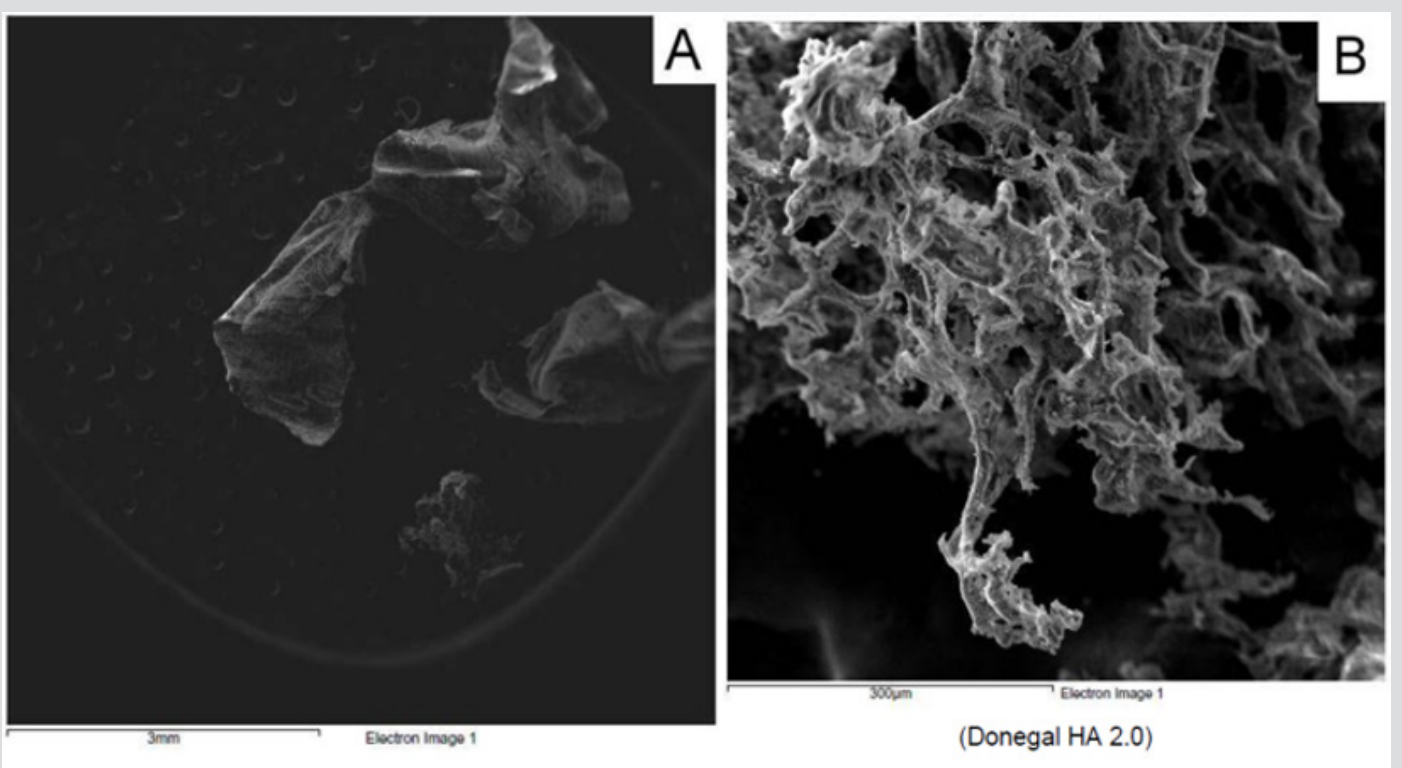

Figure 3: ESEM images of HA from formulation 1 at different magnification.

\section{Rheology Behavior}

Steady Shear Flow Experiments: In steady shear flow experiments, all samples showed a viscosity $(\eta)$ decrease when subjected to high shear strain (Figure 4A). This characteristic shear thinning behaviour classified all sample as pseudoplastic material and it is indicative of a strongly entangled polymer systems. This is the most common form of non-Newtonian behaviour, where the viscosity decreases with increasing shear rate. This is often a desirable characteristic in viscosupplementation, as it means the product can be moved around easily (pumped, spread, injected, etc.) but once it reaches its destination and comes to rest it returns to a high viscosity, which helps it maintain its position. In particular, a marked difference in viscosity at low shear rate (ẙ) is appreciable between samples, while at high shear rate values tend to approach each other. From the Figure 4A, we can see how HA formulation 1 has the highest viscosity at low shear rate, while the $2^{\text {nd }}$ formulation shows the lowest. Moreover, the sample tends to a Newtonian plateau with decreasing shear rate. The plateau viscosity, known as the zero-shear viscosity, is a useful and important material attribute, signifying the effective viscosity in an at-rest condition. 
Since the magnitude of the zero-shear viscosity is influenced by molecular weight, from Figure 4A, we can see how HA formulation 1 has, probably, the highest molecular weight, while HA from manufacturer 2 has the lowest. Furthermore, it is interesting to note how the flow curves of formulation 1 crossed the curve of formulation 3 at $8 \mathrm{~s}^{-1}$, showing a faster viscosity decrease at high shear rate than HA formulation 3.
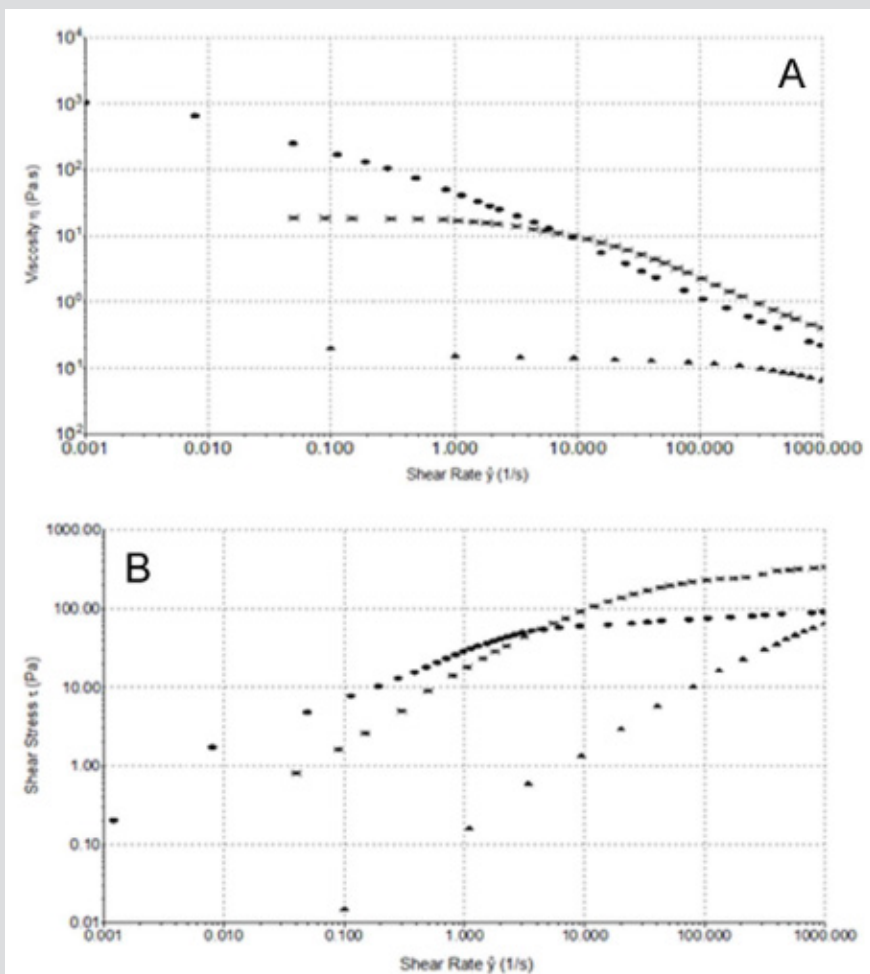

Figure 4: A) Shear thinning behavior $\eta[\mathrm{Pa} s]$ versus $\mathrm{y}[1 / \mathrm{s}]$ at $25^{\circ} \mathrm{C}$. Formulation $1(\bullet), 2(\boldsymbol{\Delta})$ and $3\left(^{*}\right)$. B) Shear thinning behavior $\tau$ versus ẙ $\left(1000 \mathrm{~s}^{-1}\right)$ at $25^{\circ} \mathrm{C} .1(\bullet), 2(\boldsymbol{\Delta})$ and $3\left(^{*}\right)$.
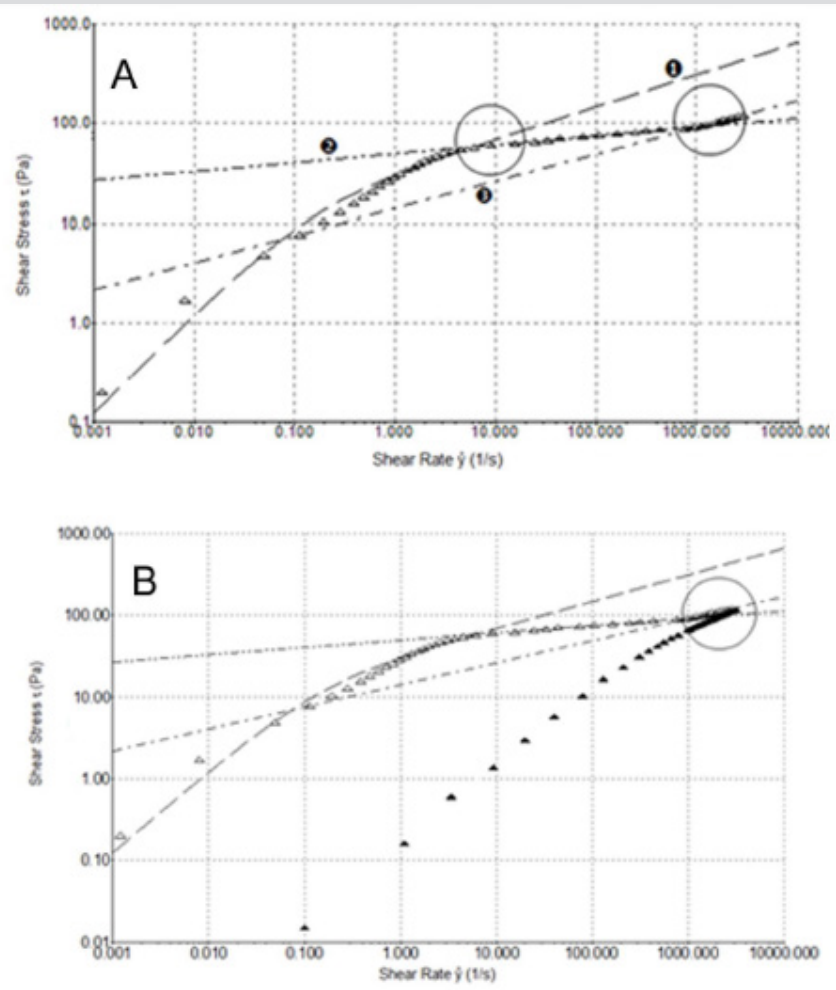

Figure 5: A) Shear thinning behavior $\tau$ versus ẙ (3000 s-1) at $25^{\circ} \mathrm{C}$ for HA from formulation 1 . B) Shear thinning behavior $\tau$ versus y $\left(3000 \mathrm{~s}^{-1}\right)$ at $25^{\circ} \mathrm{C}$ for HA formulations 1 and $2.1(\Delta)$ and $2(\boldsymbol{\Delta})$. 
In order to better understand the shear thinning behaviour of the samples, shear stress $(\tau)$ versus shear rate $(\stackrel{\circ}{)})$ plots were analyzed (Figure 4B). From the figure, we can see that formulation 1 flow curve presents a marked variation from its shear thinning behaviour at $5 \mathrm{~s}^{-1}$. Furthermore, regarding a wider shear rate range, up to $3000 \mathrm{~s}^{-1}$ (Figure 5A), it can be pointed out a second variation point at $1000 \mathrm{~s}^{-1}$. Figure $5 \mathrm{~A}$ shows regressions which approximates the experimental data in the various considered intervals $(0.001 \div 10$; $10 \div 1000 ; 1000 \div 3000 \mathrm{~s}^{-1}$ ). In Figure 5B, we compare the flow curve of HA from formulations 1 and 2 in a shear rate range up to 3000 $\mathrm{s}^{-1}$. It is worth of note that above $1000 \mathrm{~s}^{-1}$ the two flow curves tend to overlap. Despite the higher viscosity of formulation 1 compared to the other two at slow shear rates, with the increasing shear rate the viscosity of formulation 1 has a sudden decrease that modify its shear thinning behaviour at two different points and even get to cross the curve of formulation 2 who shows the lowest viscosity at low shear rate. This behaviour of formulation 1 is probably attributable to its composition formed from a hydrogel and a dispersion of HA: up to $1000 \mathrm{~s}^{-1}$ its rheological behaviour is controlled from the gel while after this value it behaves more like a HA solution. Until now, all experiments have been performed at room temperature $\left(25^{\circ} \mathrm{C}\right)$ but it is important to consider also the relationship between temperature and viscosity of these formulations. In fact, viscosity is generally inversely related with temperature. Since the final use of these formulations is viscosupplementation, we have evaluated a possible temperature involvement in the rheological behaviour at room and body temperature $\left(25^{\circ} \mathrm{C}\right.$ and $\left.37^{\circ} \mathrm{C}\right)$. We appreciated a slight viscosity decrease at the temperature rising, but the overall behaviour of the sample does not change (Supplemental Figure 1).
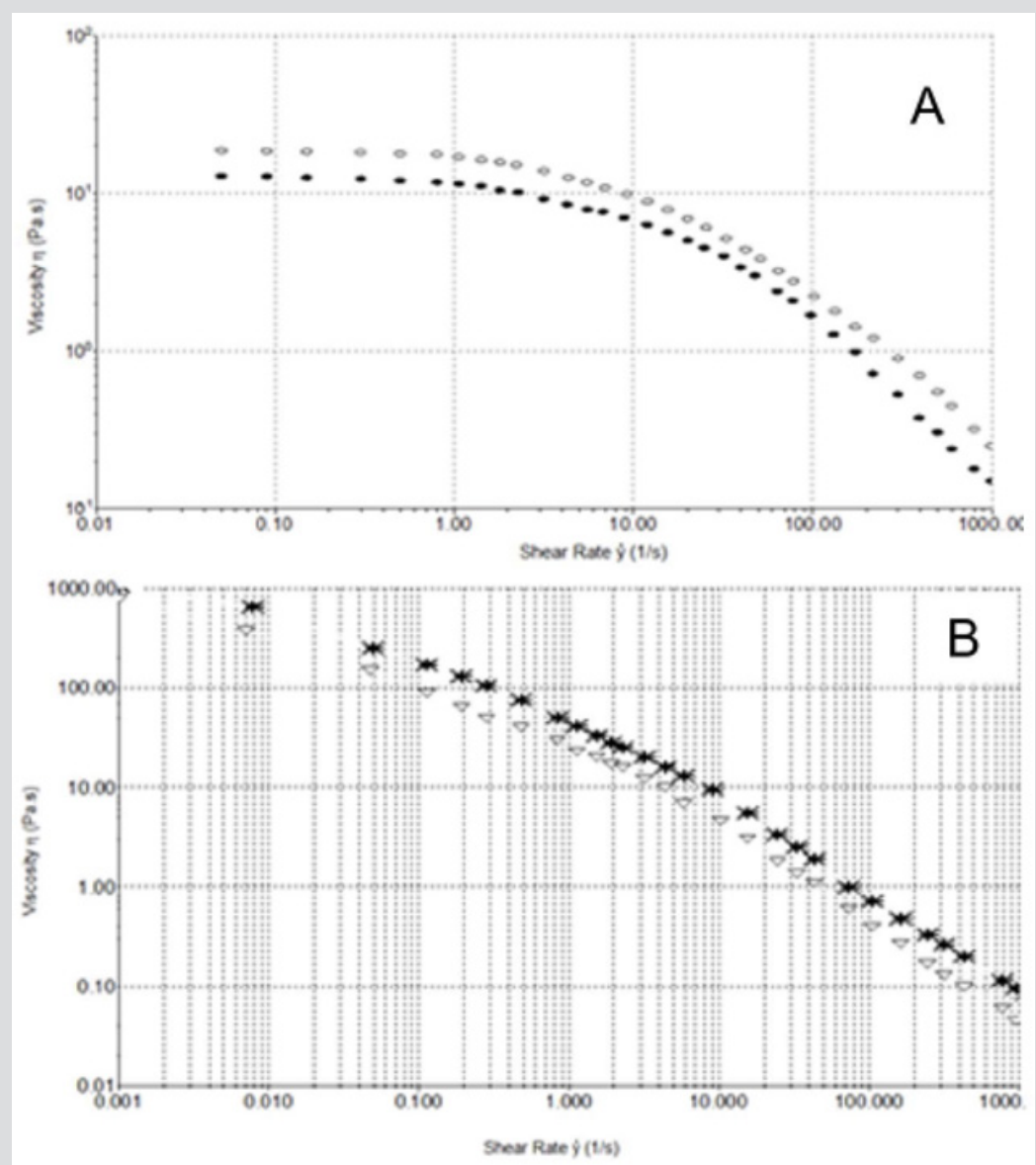

Supplemental Figure 1: A) Shear thinning behavior of formulation 3 at $25^{\circ} \mathrm{C}$ and $37^{\circ} \mathrm{C} .25^{\circ} \mathrm{C}(\mathrm{O})$ and $37^{\circ} \mathrm{C}(\bullet)$. B) Shear thinning behavior of formulation 1 at $25^{\circ} \mathrm{C}$ and $37^{\circ} \mathrm{C} .25^{\circ}\left(^{*}\right)$ and $37^{\circ} \mathrm{C}(\nabla)$.

Oscillation Stress Sweeps Experiments: In order to understand the soft-solid rigidity and gel strength of the samples, stress-sweep tests at constant frequency of $1 \mathrm{~Hz}$ were performed. HA from formulations 1 and 3 showed the independence of elastic storage modulus G' from the oscillatory stress, ranging from 0.1 to $10 \mathrm{~Pa}$. Notably, for HA formulation 2 a ranging of independence of $\mathrm{G}^{\prime}$ from the stress is observed from 0.1 to $1 \mathrm{~Pa}$, a much more limited region (Figure 6A). Figure 6B illustrates a wider range of oscillatory stress, up to $250 \mathrm{~Pa}$, to better analyze HA formulations 1 and 3 behaviour. We can see how at $90 \mathrm{~Pa}$ the $\mathrm{G}^{\prime}$ modulus of formulation 1 become lower than $3 G^{\prime}$ modulus. The greater value of ' $\mathrm{G}^{\prime}$ of formulation 1 at low oscillatory stress is the result of the enhanced solid-like character of this viscosupplement due to the presence of covalent cross-links, which increased the stiffness and show a prevailing physical hydrogel nature. Instead, while formulation 3 shows a lower G' modulus than formulation 1, the wider independence region from the stress indicates a softer but stronger formulation, probably due to the high HA concentration in formulation 3. On the contrary, behaviour of the formulation 2 is due to a low HA concentration. The linearity of this behaviour is furthermore confirmed by $\tau$ versus $\gamma$ plots (Supplemental Figure 2). 


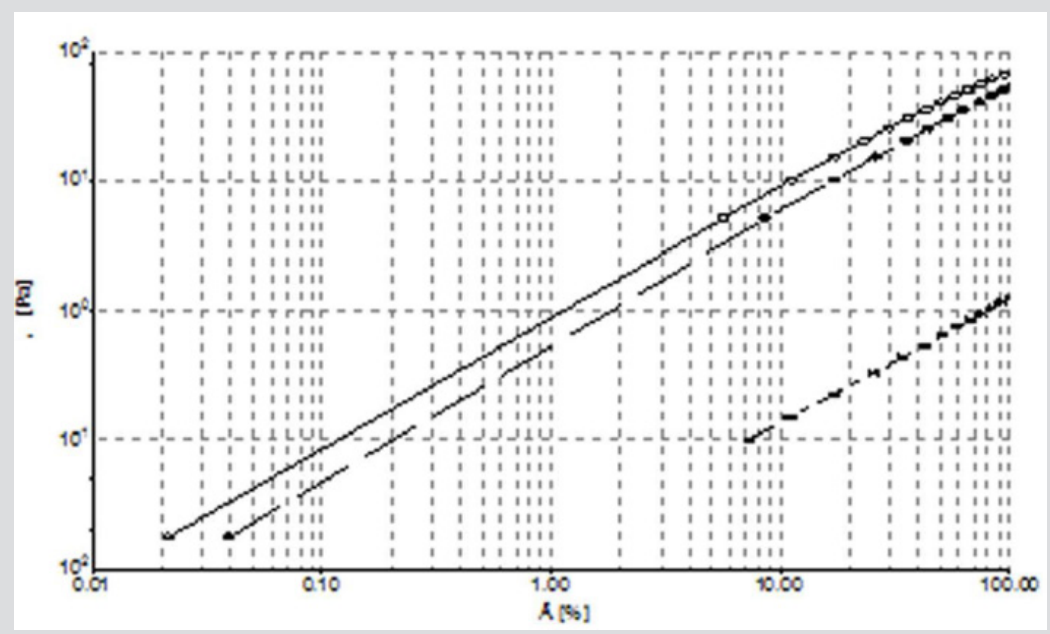

Supplemental Figure 2: $\tau$ versus $\gamma$ for $1 \mathrm{~Hz}$ at $25^{\circ} \mathrm{C}$. Formulation $1(\mathrm{o}), 2(*)$, and $3(\bullet)$ and $3(*)$.
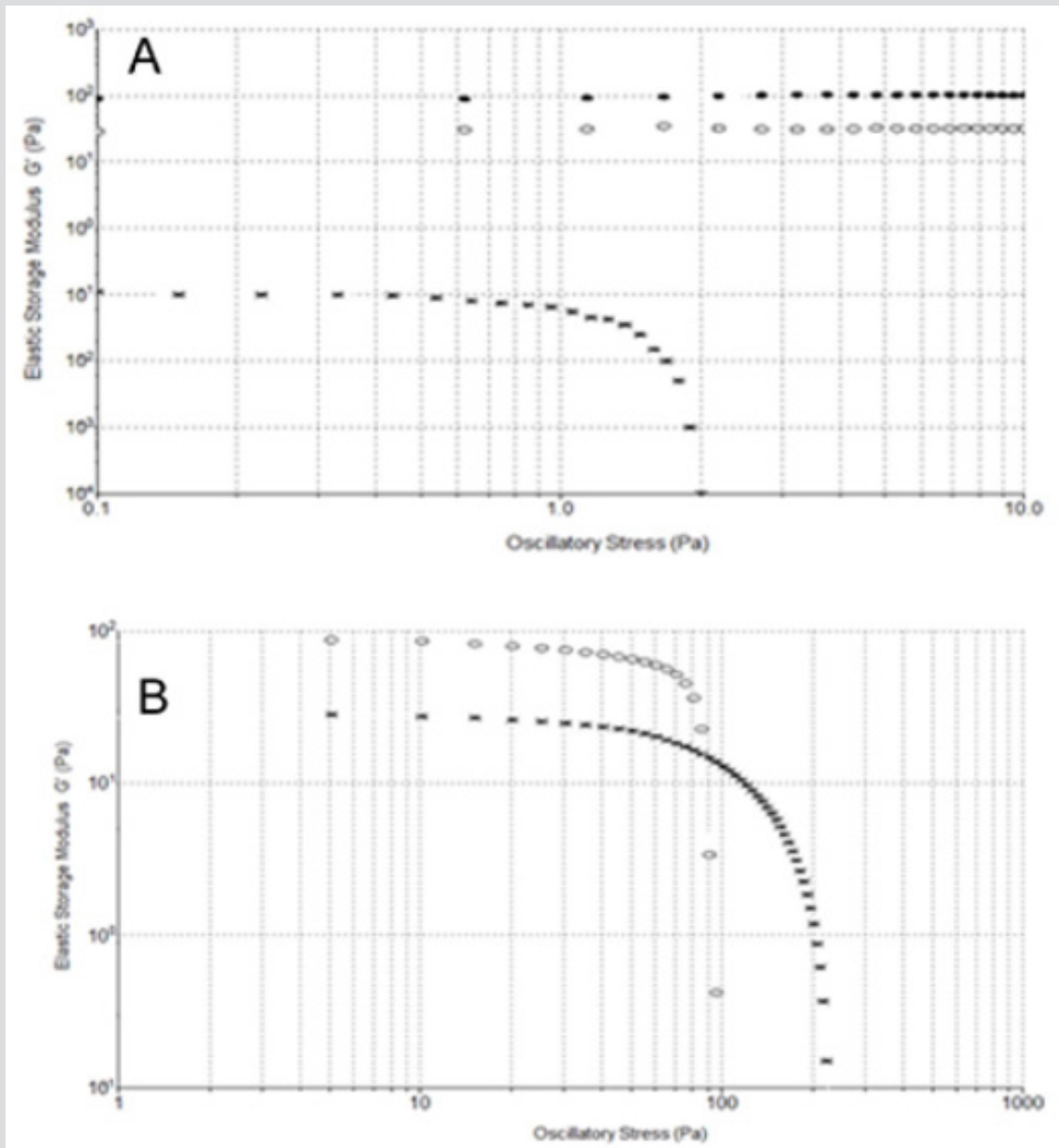

Figure 6: A) Stress-sweep at constant frequency of $1 \mathrm{~Hz}$ at $25^{\circ} \mathrm{C}$. Formulation $1(\cdot), 2\left(^{*}\right)$ and 3 (o). B) Stress-sweep at constant frequency of $1 \mathrm{~Hz}$ at $25^{\circ} \mathrm{C}$. Formulation 1 (o) and $3\left(^{*}\right)$.

Oscillatory Frequency Sweeps Experiments: The viscoelastic behaviour of HA from formulations 1 and 3 was tested in oscillatory frequency sweeps at stress of $1 \mathrm{~Pa}$, while from formulation 20.1 PA stress were used. The viscoelastic behaviour of a system is characterized by the storage modulus, G', and the loss modulus, G', which respectively characterize the solid-like and fluid-like contributions to the measured stress response. Figure 7A shows the storage and loss moduli of formulation 1 and 3 as a function of frequency at $25^{\circ} \mathrm{C}$, while Figure $7 \mathrm{~B}$ illustrates the storage and loss moduli of formulation 2 at $0.1 \mathrm{~Pa}$ as a function of frequency at $25^{\circ} \mathrm{C}$. The vanished region indicates the frequency of a knee joint corresponding to a walk $(0.5 \mathrm{~Hz}$, lower limit) and to a run ( $3 \mathrm{~Hz}$, higher limit), respectively. 

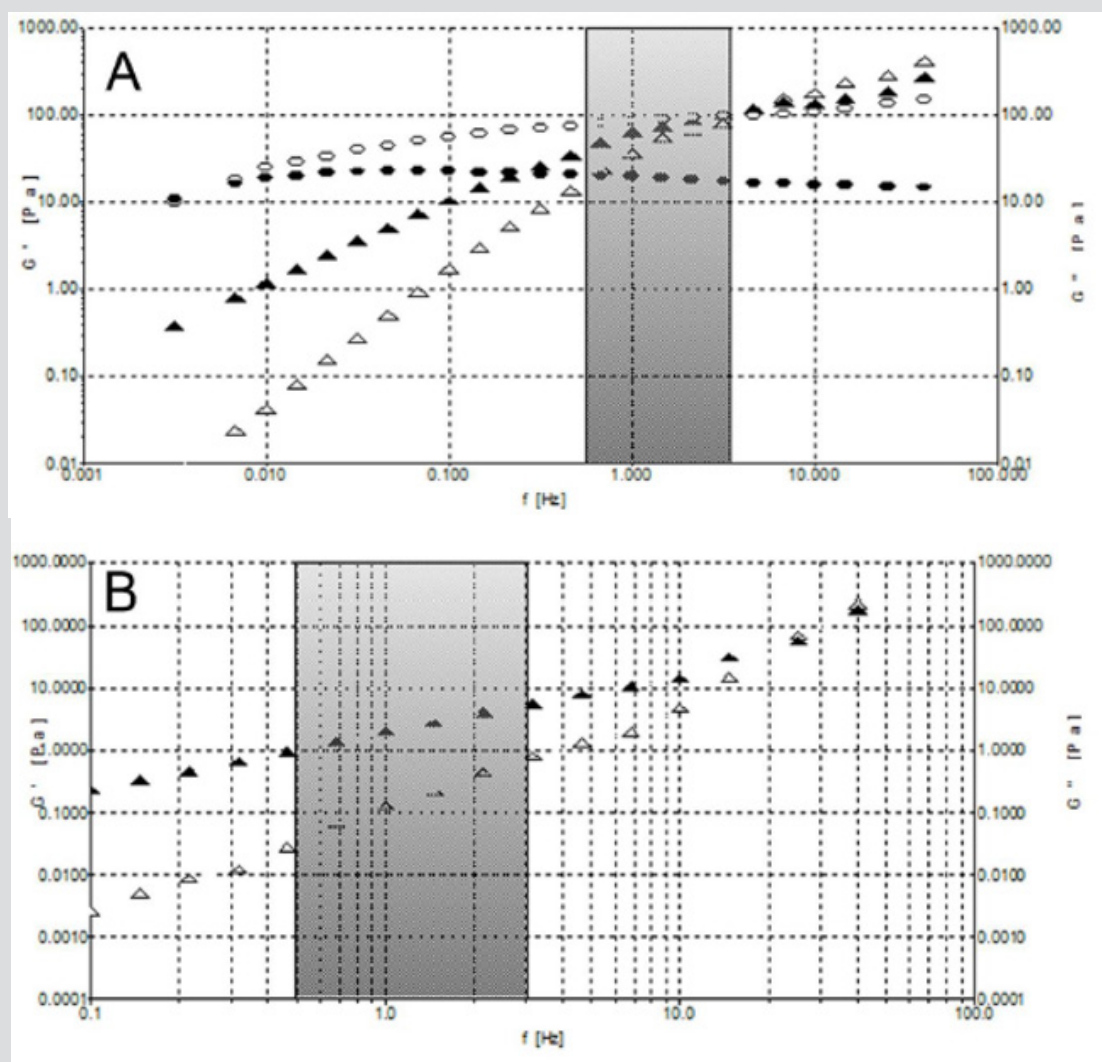

Figure 7: A) Frequency sweeps at stress of $1 \mathrm{~Pa}$ at $25^{\circ} \mathrm{C}$. Formulation $1: \mathrm{G}^{\prime}(\mathrm{o})$ and $\mathrm{G}^{\prime \prime}(\bullet)$. Formulation $3: \mathrm{G}^{\prime}(\triangle)$ and $\mathrm{G}^{\prime \prime}(\mathbf{\Delta})$. B) Frequency sweeps at stress of $0.1 \mathrm{~Pa}$ at $25^{\circ} \mathrm{C}$ for formulation 2: $\mathrm{G}^{\prime}(\triangle)$ and $\mathrm{G}^{\prime \prime}(\mathbf{\Delta})$.

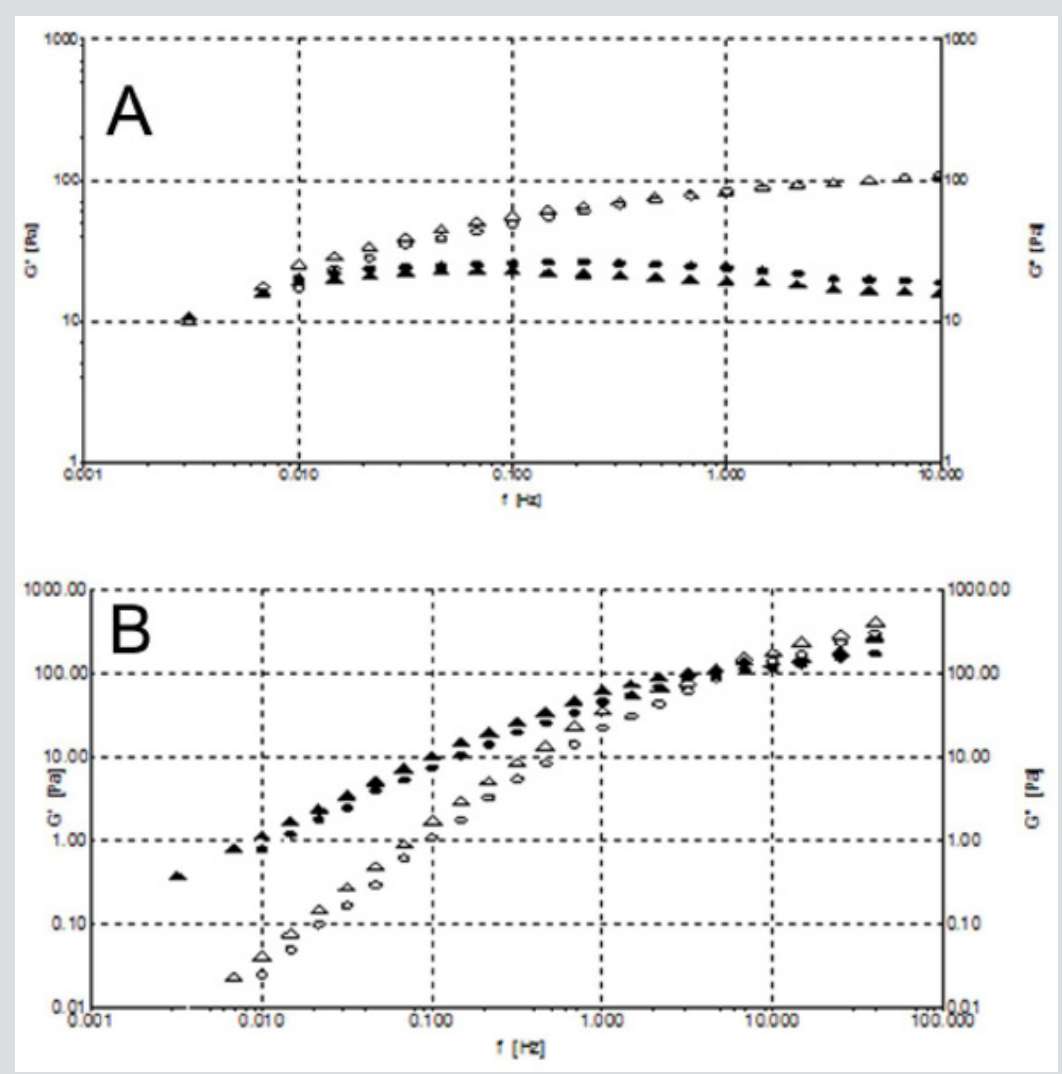

Supplemental Figure 3: A) Frequency sweeps at stress of $1 \mathrm{~Pa}$ at $25^{\circ} \mathrm{C}$ and $37^{\circ} \mathrm{C}$ for formulation $1: 25^{\circ} \mathrm{C} \mathrm{G}^{\prime}(\triangle), \mathrm{G}^{\prime \prime}(\mathbf{\Delta})$ and $37^{\circ} \mathrm{C} \mathrm{G}^{\prime}(\mathrm{o}), \mathrm{G}^{\prime \prime}(\bullet)$; B) Frequency sweeps at stress of $1 \mathrm{~Pa}$ at $25^{\circ} \mathrm{C}$ and $37^{\circ} \mathrm{C}$ for formulation 3:. $25^{\circ} \mathrm{C} \mathrm{G}^{\prime}(\triangle), \mathrm{G}^{\prime \prime}(\boldsymbol{\Delta})$ and $37^{\circ} \mathrm{C}$ $\mathrm{G}^{\prime}(\mathrm{o}), \mathrm{G}^{\prime \prime}(\bullet)$. 
Moreover, we analyzed the storage and loss moduli of formulations 1 and 3 as a function of temperature at $25^{\circ} \mathrm{C}$ and $37^{\circ} \mathrm{C}$ (Supplemental Figure 3). We can observe how only HA from formulation 1 displays viscoelastic properties typical of a gel, with $G^{\prime}>G^{\prime \prime}$, in the frequency region of interest. In fact, the crossover point for formulation 1 , the frequency $(\omega c)$ at which $G^{\prime}=G^{\prime \prime}$, falls well below the range of frequency at which the knee usually works. Instead, formulations 2 and 3 display a solution behaviour, with G'> G', in the investigated frequency range. However, unlike formulation 2, formulation 3 crossover point falls in the middle of Table 2: Crossover points of samples at $25^{\circ} \mathrm{C}$ and $37^{\circ} \mathrm{C}$. the frequency range of interest showing a considerable rheological behaviour. Interestingly, the crossover points of the examined samples clearly reveal their different rheological behaviour allowing the investigated materials to be sorted into two classes, gel-like material with lower $\omega c$ values and solution-like materials placed with higher $\omega c$ (Table 2). In all formulations, the increase of temperature is directly proportional to the increase of frequency, with a higher G' modulus and lower G" modulus. Anyway, even at $37^{\circ} \mathrm{C}$, HA from formulation 1 is the only one to show the required viscoelastic properties.

\begin{tabular}{|c|c|c|}
\hline HA Formulation & $\mathbf{\Omega c}(\mathbf{H z})$ at $\mathbf{2 5}{ }^{\circ} \mathbf{C}$ & $\mathbf{\Omega c}(\mathbf{H z})$ at $\mathbf{3} 7^{\circ} \mathbf{C}$ \\
\hline 1 (Synvisc) & 0.006 & 0.014 \\
\hline 2 (Hyalgan) & 21.5 & 28.0 \\
\hline 3 (Donegal HA 2.0) & 4.6 & 7.3 \\
\hline
\end{tabular}

Creep and Recovery Experiments: Creep-recovery is a time dependent rheological experiment in which a constant shear stress is applied at sample until it reaches the so-called creep time. After this, the stress is removed and the shear recovery is measured during the recovery time. Creep-recovery experiment has been performed to quantify the percentage of elastic recovery and to evaluate the mechanical response to the creep. Results showed for formulation 1 an evident recovery of about 30\% in the recovery phase (Figure $8 \mathrm{~A}$ ) with a viscoelastic gel typical response. Figure $8 \mathrm{~B}$ illustrates the creep and recovery of formulation 3 showing an extremely limited recovery, in order of 1\% typical of a Maxwell model belonging to a viscoelastic fluid.
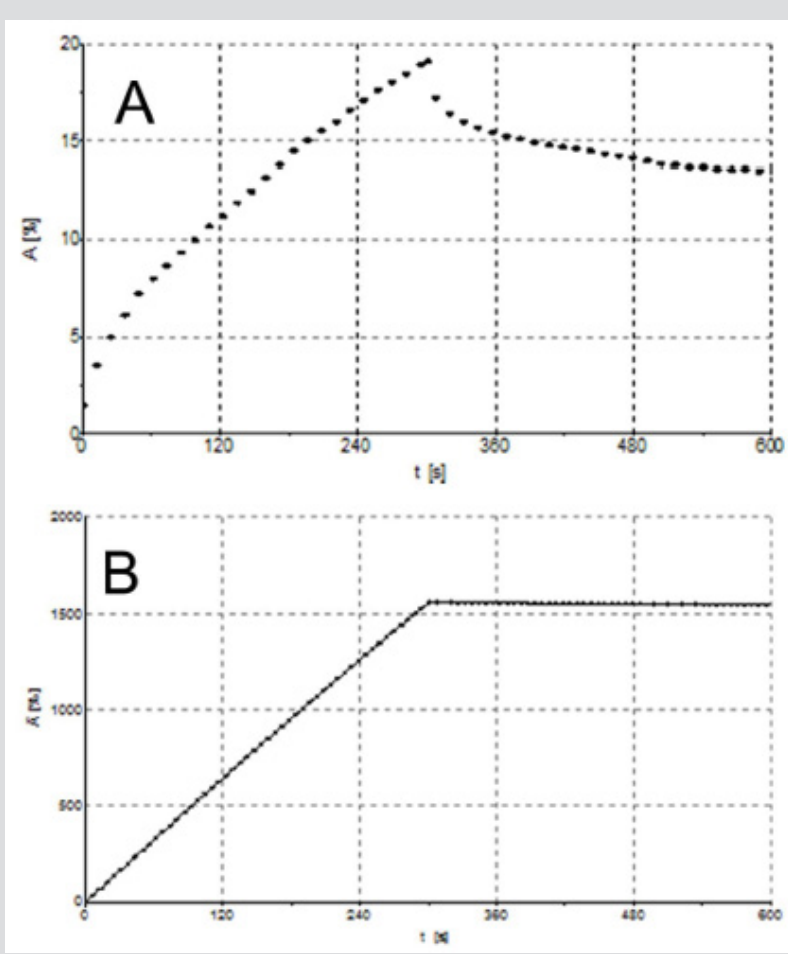

Figure 8: A) Creep and recovery for formulation 1 at $1 \mathrm{~Pa}$ and $25^{\circ} \mathrm{C}$. B) Creep and recovery for formulation 3 at $1 \mathrm{~Pa}$ and $25^{\circ} \mathrm{C}$.

\section{Inhibition of MMP-2 and MMP-9 Activity}

Formulation 1 was able to inhibit the activity of MMP-2 $72 \mathrm{kDa}$ of $\sim 21 \%$ and sample 2 of $\sim 32 \%$. Formulation 3 was found more effective with an inhibition of $\sim 48 \%$. This activity was determined to be significantly higher $(\mathrm{p}<0.001)$ than that measured for formulations 1 and 2 (Figure 9). Formulation 1 was also able to inhibit the activity of MMP-9 complexed forms at $225 \mathrm{kDa}$ of $\sim$ $13 \%$ and formulation 2 of $\sim 39 \%$. Formulation 3 was again found more effective with an inhibition of $\sim 100 \%$. This activity was significantly higher $(\mathrm{p}<0.001)$ than that measured for formulations 1 and 2 (Figure 9). Contemporary, formulation 1 was able to inhibit the activity of MMP-9 $92 \mathrm{kDa}$ of $\sim 66 \%$ and sample 2 of $\sim 24 \%$. Formulation 3 was found more effective with an inhibition of $\sim 82 \%$. This activity was again determined to be significantly higher $(p<0.001)$ than that measured for the other two formulations (Figure 9). 


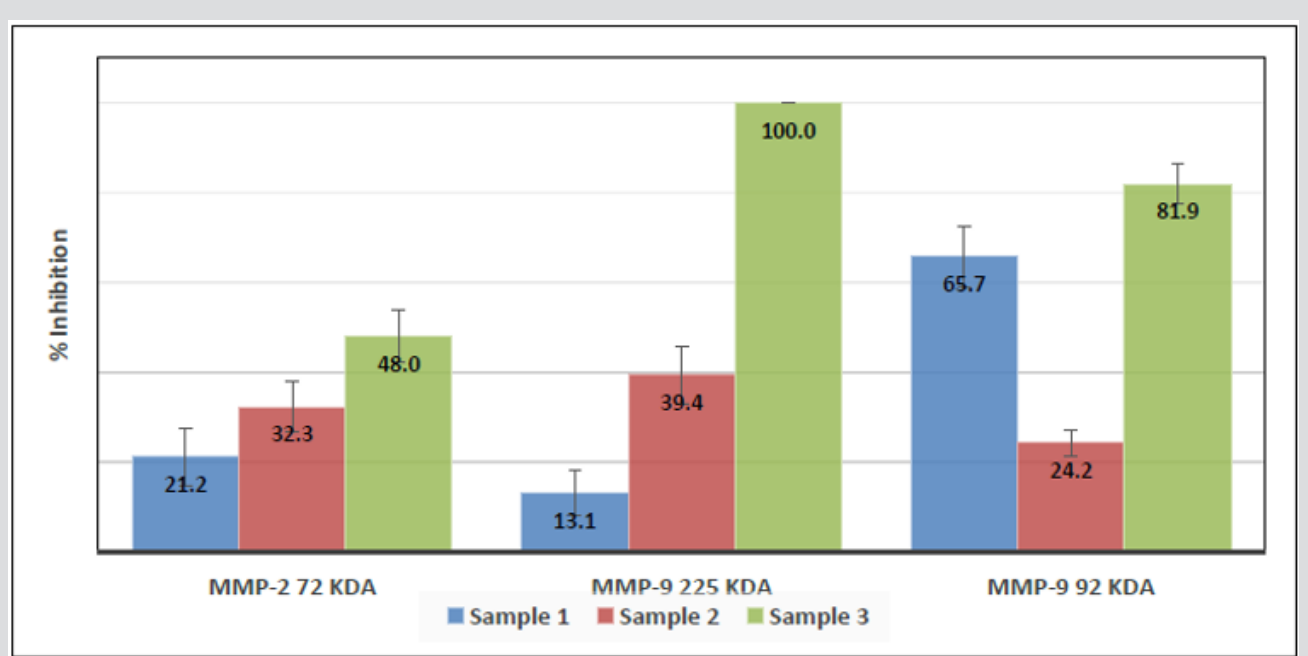

Note: Data are means with SD in bars: Sample 1 = Formulation 1 (Synvisc); Sample 2 = Formulation 2 (Hyalgan); Sample 3 = Formulation 3 (Donegal HA 2.0).

Figure 9: MMP-2 72 kDa, MMP-9 complexed forms at $225 \mathrm{kDa}$ and MMP-9 $92 \mathrm{kDa}$ percentage of inhibition by the three HA formulations.

\section{Discussion}

In steady shear flow experiments, all HA formulations showed a characteristic shear thinning behaviour that classify all of them as pseudoplastic material. Regarding formulation 1, we demonstrate how it presents the higher viscosity at low shear rate probably due to its cross-linked nature and high molecular weight. However, at high shear rate, this formulation presents a marked variation of its rheological behaviour. In fact, after $1000 \mathrm{~s}-1$, it looks more like a HA solution and not a gel. The presence of covalent cross-links in this HA formulation is clearly evidenced by the ESEM analysis in which we can see a filamentous structure that tend to be distributed side by side in longitudinal mode. This particular organization probably increases the stiffness and shows a prevailing physical hydrogel nature. In fact, this formulation shows a great value of G' modulus at low oscillatory stress, but even if it has a wider independence from the stress than the formulation 2, it is not as stronger as observed for formulation 3. According to its composition, formulation 1 is the only sample showing viscoelastic properties typical of a gel, with $\mathrm{G}^{\prime}$ $>G^{\prime \prime}$, in the frequency region in which the knee works, furthermore confirmed from the creep and recovery test in which there is a viscoelastic gel typical response, with an evident recovery of about $30 \%$ in the recovery phase. Anyway, it has been showed that crosslinked HA preparations, like formulation 1, could have increased immunogenicity attributable to the subsequent chemical crosslinking process able to enhance the average molecular weight. These polymers are often generated by chemical modification of HA samples involving formaldehyde and vinylsulfone processes.

The resulting bridges and sulfonyl-bis-ethyl cross-links generate covalently linked three-dimensional secondary and tertiary structures in these polymers which are far more complex than any seen in not modified HA. The size and complexity of this structure are likely to result in novel antigenic determinants that are not present in naturally-derived not cross-linked HA products [27]. From a clinical perspective, a published review and metanalysis [28] has concluded that, given the likely lack of a superior effectiveness of hylan over HAs and the increased risk of local adverse events associated with hylan, the use of i.a. hylan in patients with knee Osteoarthritis $(\mathrm{OA})$ in clinical practice should be discouraged. Hyaluronic acid from formulation 2 shows instead the lowest viscosity at low shear rate, the lowest independence from the oscillatory stress and a rheology behavior more like a solution material, with G'>G'. From ESEM analysis, we can see how the filaments of these samples tend to clump together without a clear and repeated organization. The lack of an organized structure is probably the reason of the weaker gel behaviour of this formulation. Formulation 3, as the above reported formulation 2, shows a lower viscosity and a lack of gel-like behaviour compared to formulation 1.

However, this HA formulation has a wider independence region from the oscillatory stress indicating a softer but stronger formulation. Moreover, the ESEM analysis shows a pseudo-spongy thick filaments where HA is distributed in a compact branched nature suggesting that this macromolecule has the strong ability to interact with a great content of water. Rheumatoid Arthritis (RA) and OA are characterized by an irreversible destruction of the cartilage, tendon, and bone that surround the synovial joints. In both RA and OA, inflammatory cytokines and tumor necrosis factor-alpha stimulate the production of MMPs, which can degrade all components of the ECM. The collagenases, MMP-1 and MMP-13 are rate limiting in the process of collagen degradation in these diseases. Expression of other MMPs such as MMP-2 and MMP-9 is also elevated in arthritis. They can degrade not only collagen such as type IV collagen in basement membrane, but also the noncollagen components of the joints [29]. Research has been made to develop effective inhibitors of MMP activity to counteract the destruction of connective tissues. Finally, by considering the above illustrated results, formulation 3 (Donegal HA 2.0), along with its rheology behaviour, capacity to interact with a great content of water and non-immunogenic properties, shows the higher ability in inhibiting MMP-2 and MMP-9 activity compared to the other two 
formulations, probably due to its specific molecular weight and/or higher HA concentration, making it a more adequate product for the treatment of diseases like arthritis.

\section{Conclusion}

The three tested HA formulations differ in rheological properties and inhibition of MMP-2 and MMP-9 activity. Among the three tested formulations, formulation 3 (Donegal HA 2.0) seems to be the most appropriate one for the treatment of osteoarthritis and rheumatoid arthritis.

\section{References}

1. Fraser J, Laurent T, Laurent U (1997) Hyaluronan: Its nature, distribution, functions and turnover. J Intern Med 242(1): 27-33.

2. Lee H, Cowman M (1994) An agarose gel electrophoretic method for analysis of hyaluronan molecular weight distribution. Anal Biochem 219(2): 278-87.

3. Toole B (2001) Hyaluronan in morphogenesis. Semin Cell Dev Biol 12(2): 79-87.

4. Toole BP (2004) Hyaluronan: From extracellular glue to pericellular cue. Nat Rev Cancer 4(7): 528-539.

5. Fraser J, Laurent $T$ (1986) The properties and turnover of hyaluronan Ciba Found Symp 124: 9-29.

6. Lapcík L, Lapcik L, De Smedt S, Demeester J, Chabrecek P (1998) Hyaluronan: Preparation, structure, properties, and applications. Chem Rev 98(8): 2663-2684.

7. Cleland RL (1984) Viscometry and sedimentation equilibrium of partially hydrolyzed hyaluronate: Comparison with theoretical models of wormlike chains. Biopolymers 23: 647-666

8. Hokputsa S, Jumel K, Alexander C, Harding S (2003) Hydrodynamic characterisation of chemically degraded hyaluronic acid. Carbohydrate Polymers 52: 111-117.

9. Matteini P, Dei L, Carretti E, Volpi N, Goti A, et al. (2009) Structural behavior of highly concentrated hyaluronan. Biomacromolecules 10(6): 1516-1522.

10. Kogan G, Šoltés L, Stern R, Mendichi R (2007) Hyaluronic acid: A biopolymer with versatile physic-chemical and biological properties. In: Pethrich RA, Ballada A, Zaikov GE (Eds.), Handbook of polymers research monomers, olygomers, polymers and composites. Nova Science Publishers, New York, USA, pp. 393-439.

11. Tamer TM (2013) Hyaluronan and synovial joint: function, distribution and healing. Interdiscip Toxicol 6(3): 111-125.

12. Morris E, Rees D, Welsh E (1980) Conformation and dynamic interactions in hyaluronate solutions. J Mol Biol 138(2): 383-400.

\section{ISSN: 2574-1241}

DOI: 10.26717/BJSTR.2019.17.002938

Baraldi Enrica. Biomed J Sci \& Tech Res

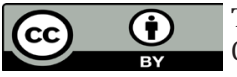

This work is licensed under Creative

Commons Attribution 4.0 License

Submission Link: https://biomedres.us/submit-manuscript.php
13. Gibbs D, Merrill E, Smith K, Balazs E (1968) Rheology of hyaluronic acid. Biopolymers 6(6): 777-791.

14. Cowman M, Matsuoka S (2005) Experimental approaches to hyaluronan structure. Biopolymers 340(5): 791-809.

15. Boettger M, Kümmel D, Harrison A, Schaible H (2011) Evaluation of long-term antinociceptive properties of stabilized hyaluronic acid preparation (NASHA) in an animal model of repetitive joint pain. Arthritis Ress Ther 13(4): R110.

16. Allison D, Grande-Allen K (2006) Review. Hyaluronan: A powerful tissue engineering tool. Tissue Eng 12(8): 2131-2140.

17. Kuo J (2006) Practical aspects of Hyaluronan based medical products. CRC Press, pp. 240.

18. Laurent T (1998) The chemistry, biology, and medical applications of hyaluronan and its derivates. Portland Press, Miami, pp. 341.

19. Zhu W, Mow V, Rosenberg L, Tang L (1994) Determination of kinetic changes of aggrecan-hyaluronan interactions in solution from its rheological properties. J Biomech 27(5): 571-579.

20. Hardingham T (2004) Chemistry and biology of hyaluronan. Elsevier, p. 1-20.

21. Xu X, Jha A, Harrington D, Farach-Carson M, Jia X (2012) Hyaluronic acidbased hydrogels: From a natural polysaccharide to complex networks. Soft Matter 8(12): 3280-3294.

22. Oh J, Drumright R, Siegwart D, Matyjaszewski K (2008) The development of microgels/nanogels for drug delivery applications. Prog Polym Sci 33(4): 448-477.

23. Balazs E, Denlinger J (1993) Viscosupplementation: A new concept in the treatment of osteoarthritis. J Rheumatol Suppl 39: 3-9.

24. Maneiro E, de Andres M, Fernandez-Sueiro J, Galdo F, Blanco F (2004) The biological action of hyaluronan on human osteoartritic articular chondrocytes: the importance of molecular weight. Clin Exp Rheumatol 22(3): 307-312.

25. Amiel D, Toyoguchi T, Kobayashi K, Bowden K, Amiel M, et al. (2003) Long-term effect of sodium hyaluronate (Hyalgan) on osteoarthritis progression in a rabbit model. Osteoarthr Cartilage 11(9): 636-643.

26. Moreland L (2003) Intra-articular hyaluronan (hyaluronic acid) and hylans for the treatment of osteoarthritis: mechanisms of action. Arthritis Res Ther 5(2): 54-67.

27. Goldberg VM, Coutts RD (2004) Pseudoseptic reactions to hylan viscosupplementation: Diagnosis and treatment. Clin Orthop Relat Res 419: 130-137.

28. Reichenbach S, Blank S, Rutjes AW, Shang A, King EA, et al. (2007) Hylan versus hyaluronic acid for osteoarthritis of the knee: a systematic review and meta-analysis. Arthritis Rheum 57(8): 1410-1418.

29. Burrage PS, Mix KS, Brinckerhoff CE (2006) Matrix metalloproteinases: Role in arthritis. Front Biosci 11: 529-5243.

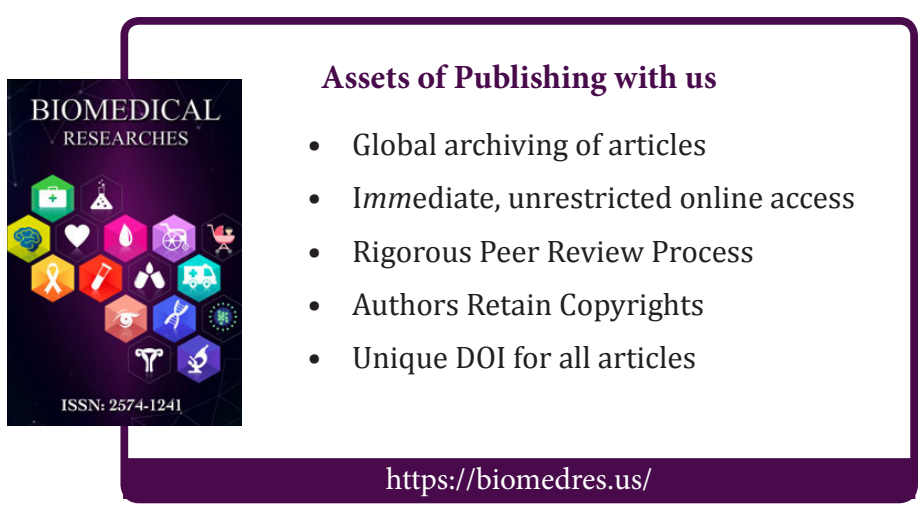

